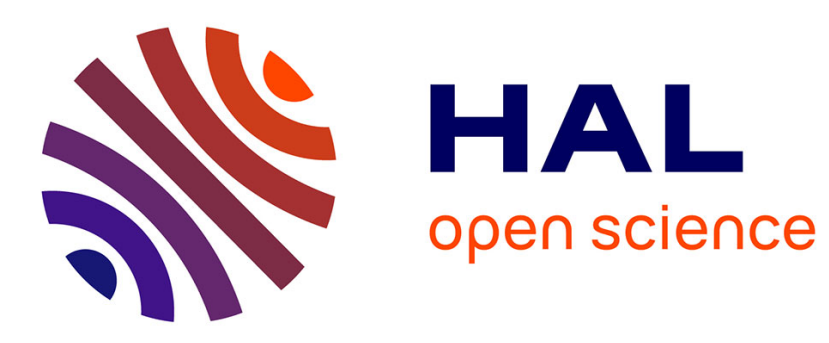

\title{
Spin glass-like behaviour in the 1D mixed compound CHAC : Mn
}

\author{
A. Cheikhrouhou, C. Dupas, J.P. Renard
}

\section{To cite this version:}

A. Cheikhrouhou, C. Dupas, J.P. Renard. Spin glass-like behaviour in the 1D mixed compound CHAC : Mn. Journal de Physique Lettres, 1983, 44 (18), pp.777-784. 10.1051/jphyslet:019830044018077700 . jpa-00232262

\section{HAL Id: jpa-00232262 https://hal.science/jpa-00232262}

Submitted on 1 Jan 1983

HAL is a multi-disciplinary open access archive for the deposit and dissemination of scientific research documents, whether they are published or not. The documents may come from teaching and research institutions in France or abroad, or from public or private research centers.
L'archive ouverte pluridisciplinaire HAL, est destinée au dépôt et à la diffusion de documents scientifiques de niveau recherche, publiés ou non, émanant des établissements d'enseignement et de recherche français ou étrangers, des laboratoires publics ou privés. 
Classification

Physics Abstracts

$75.30-75.50-75.60$

\title{
Spin glass-like behaviour in the $1 \mathrm{D}$ mixed compound CHAC : Mn
}

\author{
A. Cheikhrouhou \\ Faculté des Sciences et Techniques de Sfax, B.P.W., Sfax, Tunisia \\ C. Dupas and J. P. Renard \\ Institut d'Electronique Fondamentale $\left({ }^{*}\right)$, Université Paris XI, 91405 Orsay Cedex, France
}

(Reçu le 30 mai 1983, accepté le 29 juillet 1983)

\begin{abstract}
Résumé. - La susceptibilité alternative du composé mixte quasi-1D $\mathrm{C}_{6} \mathrm{H}_{11} \mathrm{NH}_{3} \mathrm{Cu}_{1-x} \mathrm{Mn}_{x} \mathrm{Cl}_{3}$ (CHAC : Mn) présente à $T_{\max }$ un maximum dépendant de la fréquence. Des effets de rémanence de l'aimantation apparaissent en dessous de $T_{\max }$. Les variations avec le champ, le temps et la température des aimantations thermorémanente et isotherme rémanente sont semblabes à celles des verres de spin classiques. Ces mesures suggèrent un comportement de type verre de spin dans les chaînes linéaires à échange aléatoire à très faible concentration en impuretés.
\end{abstract}

\begin{abstract}
The a.c. susceptibility of the quasi-1D mixed compound $\mathrm{C}_{6} \mathrm{H}_{11} \mathrm{NH}_{3} \mathrm{Cu}_{1-x} \mathrm{Mn}_{x} \mathrm{Cl}_{3}$ (CHAC : Mn) exhibits at $T_{\max }$ a frequency dependent maximum. Remanence effects on the magnetization appear below $T_{\max }$. The variations with field, time and temperature of the thermoremanent and isothermal remanent magnetizations are similar to those observed in classical spin glasses. These measurements suggest spin glass-like behaviour in linear chains with random exchange at very low impurity concentration.
\end{abstract}

\section{Introduction.}

Recently, the magnetic properties of systems with random exchange or anisotropy have received considerable interest. As an example, indications of a behaviour characteristic of spin glasses have just been reported in the mixed two-dimensional (2D) compounds $\mathrm{Rb}_{2} \mathrm{Mn}_{1-x} \mathrm{Cr}_{x} \mathrm{Cl}_{4}$ [1] and $\mathrm{K}_{2} \mathrm{Cu}_{x} \mathrm{Mn}_{1-x} \mathrm{~F}_{4}$ [2]. In the past few years, we have studied the magnetic properties of the mixed quasi-1D compounds TMMC : $\mathrm{Cu}$ and TMCuC : $\mathrm{Mn}$ [3], in an attempt to realize a 1D spin glass with a system of chemical formula $\left(\mathrm{CH}_{3}\right)_{4} \mathrm{NMn}_{x} \mathrm{Cu}_{1-x} \mathrm{Cl}_{3}$. Pure TMMC and TMCuC are respectively good realizations of a quasi-1D Heisenberg antiferromagnet (AFM) with $S=\frac{5}{2}$ and ferromagnet (FM) with $S=\frac{1}{2}$. We have shown that in the mixed compounds, the Cu-Mn exchange interaction is weak and ferromagnetic. Nevertheless, we did not succeed in growing single crystals of TMCuC : Mn and finally did not observe the expected behaviour.

$\left.{ }^{*}\right)$ Laboratoire associé au CNRS. 
It has been shown recently that cyclohexylammonium copper chloride $\mathrm{C}_{6} \mathrm{H}_{11} \mathrm{NH}_{3} \mathrm{CuCl}_{3}$ (CHAC) is also a good quasi-1D Heisenberg FM [4]. We report here results of a.c. susceptibility and d.c. magnetization measurements on a single crystal of CHAC : $\mathrm{Mn}(0.90 \%)$ which strongly support spin-glass-like behaviour in this system.

Previous studies on pure CHAC by other authors include crystal structure, susceptibility, magnetization [5], and specific heat [6] experiments. This salt is orthorhombic, belonging to the space group $\mathrm{P} 2{ }_{1} 2_{1} 2_{1}$, with cell parameters $a=19.441 \AA$ [5], $b=8.549 \AA$ [2] and $C=6.190 \AA$ [1]. The structure consists of bibridged linear chains of $\mathrm{CuCl}_{3}^{-}$ions parallel to the $c$ axis, hydrogen bonded together in the $b-c$ plane. The CHAC presents a strong FM coupling between $\mathrm{Cu}^{++}$ions along the chain axis : the intrachain exchange interaction obtained from the susceptibility measurements is $J / k_{\mathrm{B}}=70 \mathrm{~K}$ [5] and from the specific heat $J / k_{\mathrm{B}}=45 \mathrm{~K}$ [6]. 3D antiferromagnetic (AFM) ordering is observed at $T_{\mathrm{N}}=2.18 \mathrm{~K}$. The easy spin axis is the $b$ axis, and there is a weak FM component along the $a$ axis.

\section{Experimental techniques.}

2.1 CRYSTAL GROWTH. - The samples of CHAC : Mn have been grown by slow evaporation in an oven at $40{ }^{\circ} \mathrm{C}$ of a saturated solution of cyclohexylammonium chloride, anhydrous copper (II) chloride and anhydrous manganese (II) chloride in 1-propanol. Large single crystals are easily grown in this way. The manganese and copper concentrations in these compounds were determined by chemical analysis (microanalysis laboratory of CNRS Vernaison). The Mn impurity concentration in the crystals is smaller than in the starting solutions by a factor of about 3.

2.2 MAGNETIC MEASUREMENTS. - We measured the a.c. susceptibility of our sample by means of a mutual inductance bridge operating at low frequencies $(8 \mathrm{~Hz}-1 \mathrm{kHz})$ and with a weak excitation field (about 3 Oe peak to peak). External d.c. magnetic fields could be applied to the sample. D.c. magnetizations were obtained by a fluxmetric method in d.c. applied fields up to 500 Oe. All experiments were done in the He-4 temperature range $1.2-4.2 \mathrm{~K}$.

\section{Susceptibility experiments.}

We have measured the a.c. susceptibility $\chi^{\prime}$ versus temperature of $\mathrm{CHAC}: \operatorname{Mn}(0.9 \%)$ along the $a$ axis at various frequencies from 8.5 to $1070 \mathrm{~Hz}$, but with the same excitation field.

When decreasing temperature, the susceptibility exhibits a sharp maximum at a temperature $T_{\text {max }}$ lower than in pure CHAC. A detailed study of the variation of $T_{\text {max }}$ with manganese concentration $x$ at fixed frequency has been performed and will be reported elsewhere. When increasing the measuring frequency $v$, the maximum of $\chi^{\prime}(T)$ lowers and shifts to higher temperature (Fig. 1). We have checked that such an effect does not exist in pure CHAC. The behaviour of $T_{\max }(v)$ in CHAC : Mn is similar to that observed in metallic and insulating [7] spin glasses, which are respectively characterized by long-range and short-range magnetic interactions. Nevertheless, the variation of $T_{\max }(v)$ in CHAC : Mn cannot be fitted by an Arrhenius law : $T_{\max }^{-1}=\frac{k_{\mathrm{B}}}{E}\left(-\operatorname{Ln} v-\operatorname{Ln} \tau_{0}\right)$, neither by a Fülcher law $:\left(T_{\max }-T_{0}\right)^{-1}=\frac{k_{\mathrm{B}}}{E}\left(-\operatorname{Ln} v-\operatorname{Ln} \tau_{0}\right)$

(Fig. 2). These two laws describe the spin freezing in spin glasses as a dynamical phenomenon. In this model the freezing is a progressive blocking along an anisotropy axis of non-interacting (Arrhenius) or interacting (Fülcher) clusters whose thermal relaxation time is of the form $\tau=\tau_{0} \exp \left(E / k_{\mathrm{B}} T\right)(\mathrm{A})$ or $\tau=\tau_{0} \exp \left[E / k_{\mathrm{B}}\left(T-T_{0}\right)\right](\mathrm{F})$. If we try to fit the $T_{\max }(v)$ variation 


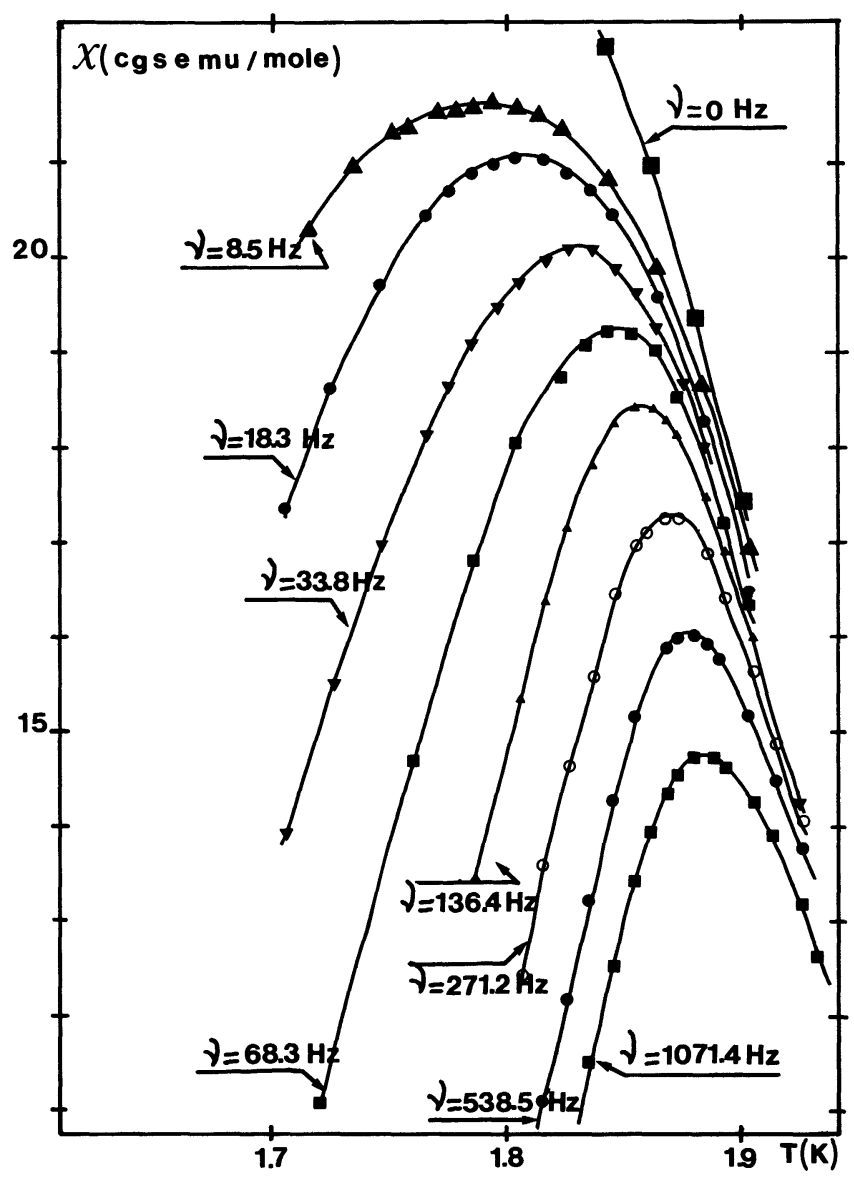

Fig. 1. - Variation with the measuring frequency of the maximum of the in-phase component $\chi^{\prime}$ of the a.c. susceptibility along the $a$ axis in the mixed compound CHAC : Mn $(0.9 \%)$. The temperature $T_{\max }$ of the maximum increases from $1.792 \mathrm{~K}$ to $1.885 \mathrm{~K}$ when $v$ increases from 8.5 to $1071.4 \mathrm{~Hz}$. Black squares correspond to the static susceptibility deduced from magnetization measurements.

in CHAC : $\mathrm{Mn}(0.90 \%)$ with an Arrhenius law, we find for $\tau_{0}$ and $E$ the clearly unphysical values $E / k_{\mathrm{B}}=195 \mathrm{~K}$ and $\tau_{0}=10^{-46} \mathrm{~s}$. Moreover, the experimental curve $T_{\max }^{-1}$ as a function of $\operatorname{Ln} v$ exhibits an upwards curvature. Anyway, experiments in a wider frequency range would be necessary before drawing any definitive conclusion.

In CHAC : Mn, we also observed that the susceptibility maximum at fixed frequency is strongly depressed by a small d.c. field ( $25 \mathrm{Oe}$ ), as in classical spin glasses (Fig. 3 ).

In order to complete these susceptibility measurements, we measured the d.c. magnetization in a weak applied field of $10 \mathrm{Oe}$ along the $a$ axis in pure $\mathrm{CHAC}$ and in $\mathrm{CHAC}: \mathrm{Mn}$. The d.c. susceptibility of pure CHAC exhibits a net plateau below $T_{\mathrm{N}}$, due to the weak FM component, as previously observed by Groenendijk et al. [5]. When doping CHAC with $\mathrm{Mn}^{++}$impurities, one obtains instead of the plateau a broad maximum whose amplitude corresponds to a lower value of the susceptibility (27.5 cgs emu/mole in CHAC : Mn against $45.5 \mathrm{cgs}$ emu/mole in pure CHAC) (Fig. 4). The weak ferromagnetism in CHAC is thus strongly perturbed by the introduction of a very low amount of impurities. A.c. and d.c. susceptibility values are found here to converge nicely at temperatures higher than $T_{\max }$. 


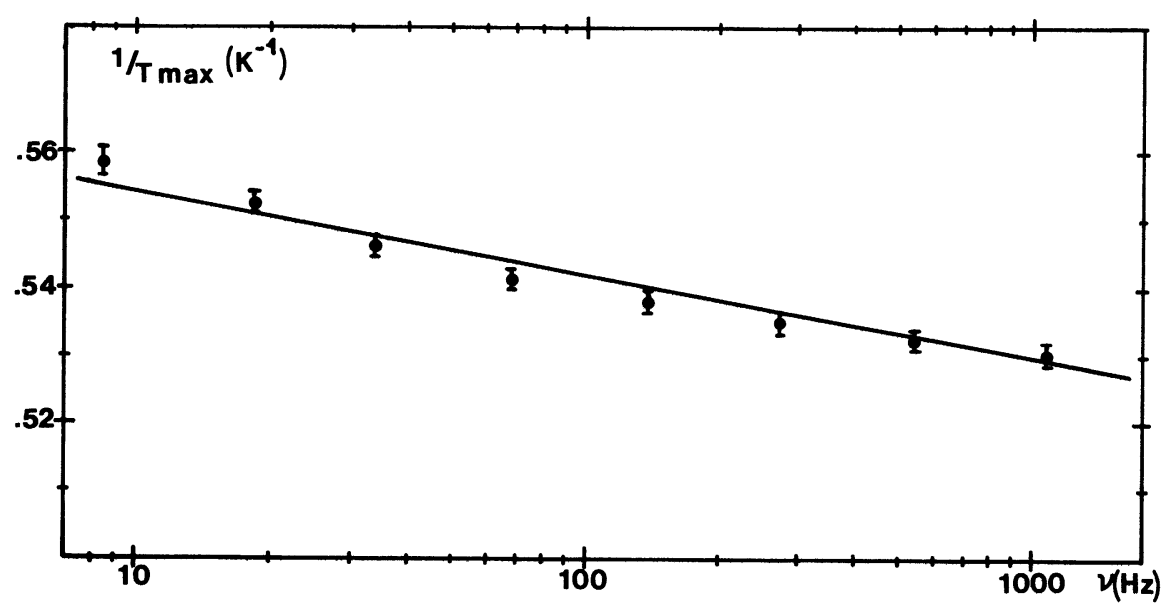

Fig. 2. - Variation of $T_{\max }^{-1}$ versus logarithm of the measuring frequency $v$. The full line corresponds to the Arrhenius law : $T_{\max }^{-1}=\frac{k_{\mathrm{B}}}{E}\left(-\operatorname{Ln} v-\operatorname{Ln} \tau_{0}\right)$ with $B / k_{\mathrm{B}}=195 \mathrm{~K}$ and $\tau_{0}=10^{-46} \mathrm{~s}$, i.e. clearly unphysical values.

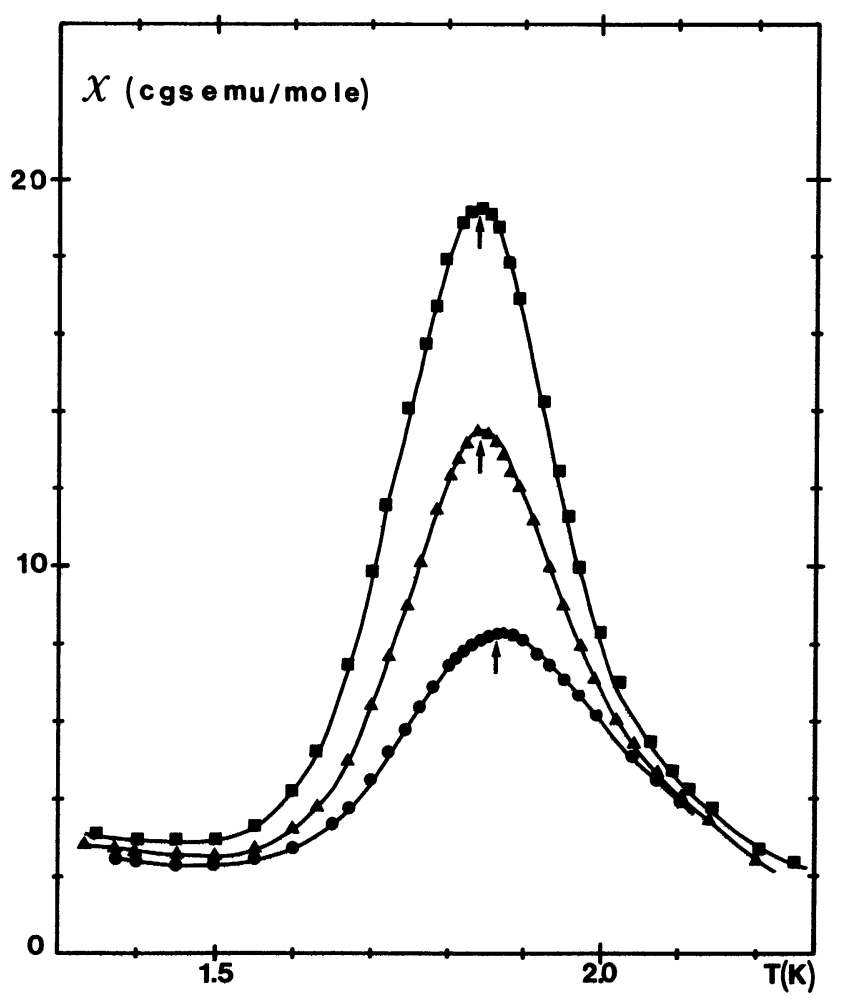

Fig. 3. - Effect of a small applied d.c. field on the susceptibility maximum measured at $68.3 \mathrm{~Hz}$ along the $a$ axis in CHAC : Mn $(0.9 \%)$. Squares : $H=0$; triangles : $H=12.3 \mathrm{Oe}$; circles $: H=25 \mathrm{Oe}$. 


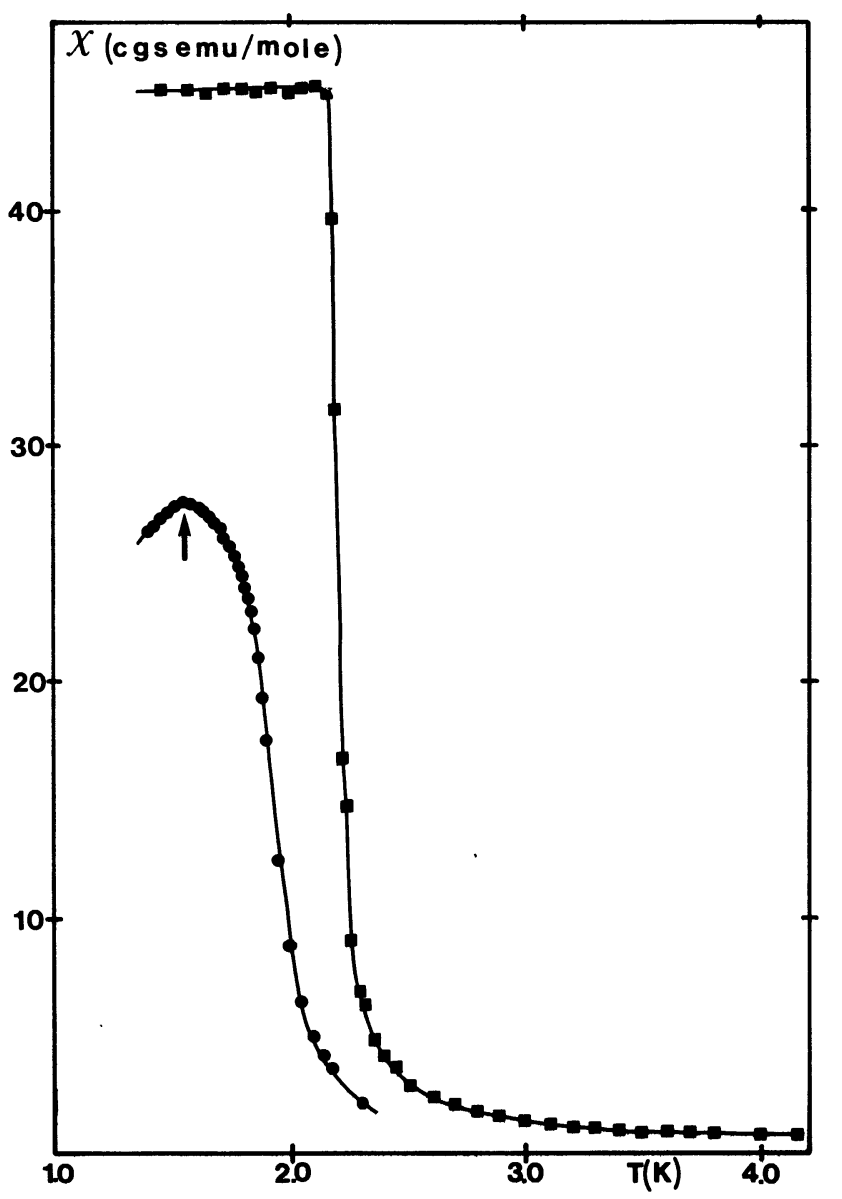

Fig. 4. - Static susceptibility $\chi=M / H$ deduced from magnetization measurements in a field $H=10 \mathrm{Oe}$ along the $a$ axis in pure CHAC (squares) and in CHAC : Mn $(0.9 \%)$ (circles). The plateau below $T_{\mathrm{N}}$ due to the weak ferromagnetic component in pure CHAC is strongly perturbed by the introduction of the Mn impurities.

\section{Remanent magnetizations.}

When switching off the d.c. field applied along the $a$ axis below $T_{\max }$ strong remanence effects appear in CHAC : Mn, which are absent in pure CHAC. As an example, the thermoremanent magnetization decreases as $\operatorname{Ln} t(t=$ time $)$ (Fig. 5). We thus performed a systematic study of the thermoremanent (TRM) and isothermal remanent (IRM) magnetizations in CHAC : Mn (0.9\%). TRM was determined as a function of field $H$ by decreasing the temperature for each fixed value of $H$ down to $1.42 \mathrm{~K}$ switching off the field and measuring the remanent magnetization after $100 \mathrm{~s}$. IRM was obtained by cooling the sample in zero field down to $1.42 \mathrm{~K}$, applying the field at that temperature during $30 \mathrm{~s}$, and measuring the remanent component $100 \mathrm{~s}$ after switching off the field. The variation with field of the TRM and IRM is very similar to that observed in spin glasses (Fig. 6). At low field, the TRM increases more rapidly than the IRM and exhibits a characteristic maximum. Both remanent magnetizations saturate at a common value, the saturated remanent magnetization (SRM), for fields higher than $40 \mathrm{Oe}$. This value of the saturation field 


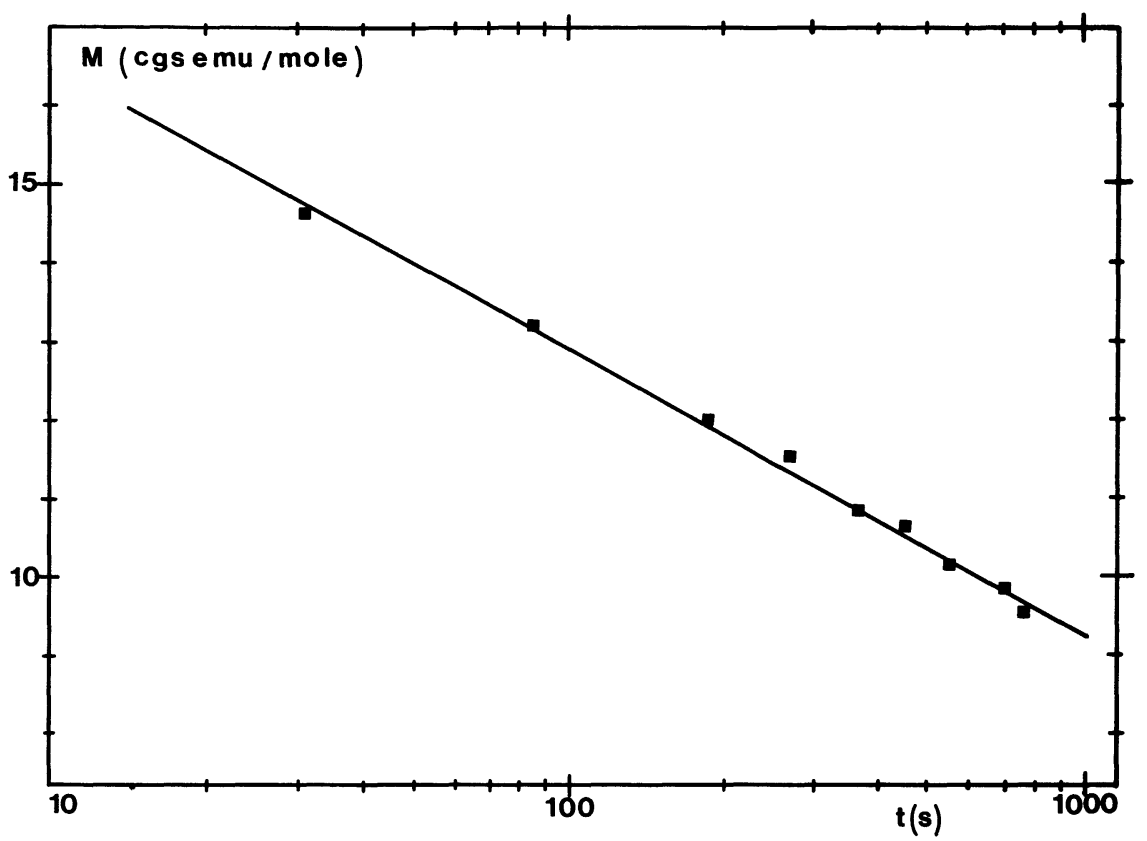

Fig. 5. - Variation of the thermoremanent magnetization along $a$ versus logarithm of the time $t$ elapsed after switching off the field $H=25 \mathrm{Oe}$. Temperature of measurement is $T=1.42 \mathrm{~K}$.

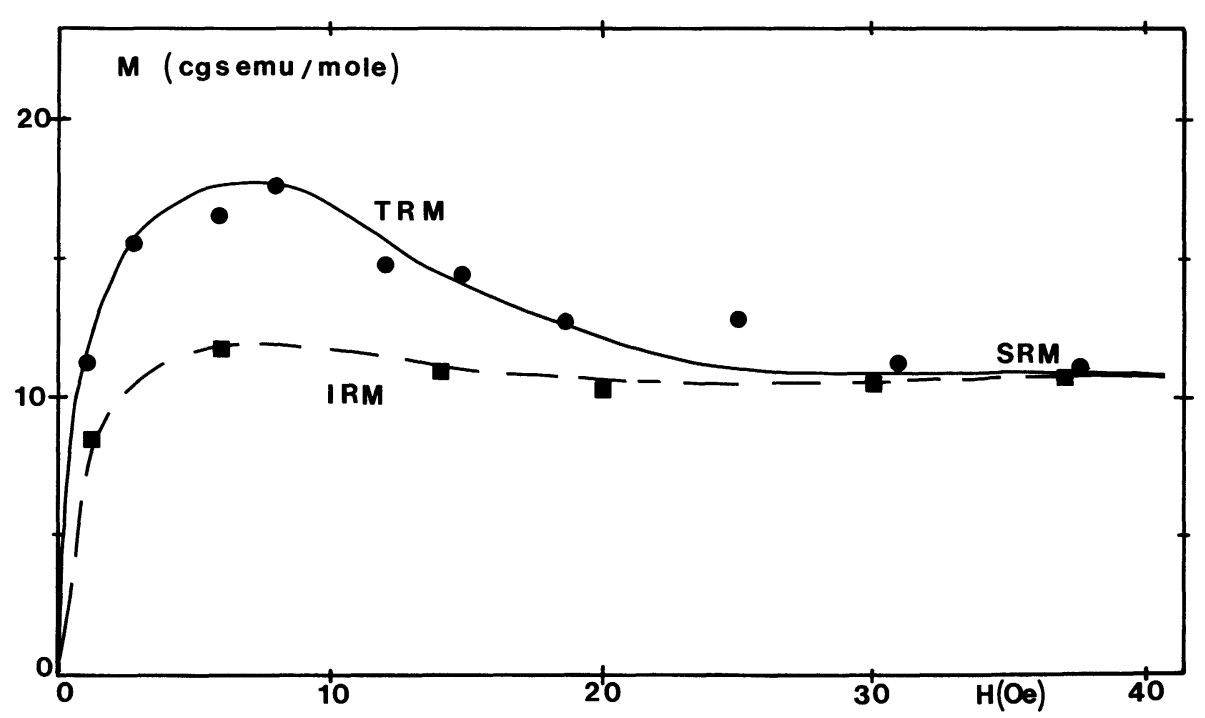

Fig. 6. - Variation with field $H$ of the thermoremanent (TRM) and isothermal remanent (IRM) magnetizations of CHAC : $\mathrm{Mn}(0.9 \%)$ along $a$ at $T=1.42 \mathrm{~K}$. Measurements were done $100 \mathrm{~s}$ after switching off the field. Saturated remanent magnetization (SRM) is obtained for $H \simeq 40 \mathrm{Oe}$.

$H_{\mathrm{s}}$ is much lower than those commonly observed in archetypal spin glasses (for example $H_{\mathrm{s}}=$ $1000 \mathrm{Oe}$ in holmium aluminosilicate [8] and $50 \mathrm{kOe}$ in CuMn $8 \%$ [9]). The amplitude of the SRM is of the order of $1 \%$ of the weak FM component. Its variation with temperature is linear (Fig. 7) 


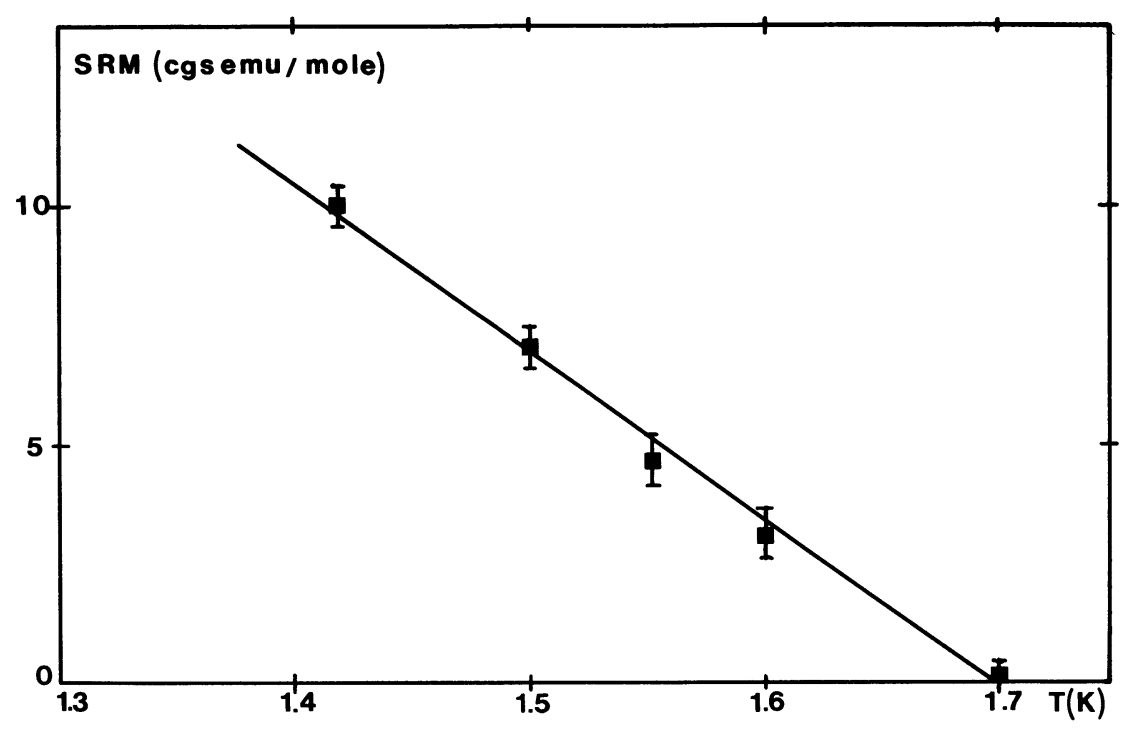

Fig. 7. - Variation with temperature of the saturated remanent magnetization (SRM) in CHAC : Mn $(0.9 \%)$ measured $100 \mathrm{~s}$ after switching off the field along $a$.

and extrapolates to zero at $T=1.7 \mathrm{~K}$. This value, corresponding to the freezing temperature for a measurement time of $100 \mathrm{~s}$, i.e. a frequency of $10^{-2} \mathrm{~Hz}$, is well situated between $T_{\max }($ d.c. $)=$ $1.55 \mathrm{~K}$ and $T_{\max }(8.5 \mathrm{~Hz})=1.79 \mathrm{~K}$. It can be noted that the temperature $T=1.42 \mathrm{~K}$ at which we have done the IRM and TRM experiments is relatively near $T_{\max }\left(10^{-2} \mathrm{~Hz}\right)=1.7 \mathrm{~K}$, the ratio being $0.84 . T=1.42 \mathrm{~K}$ was the lowest temperature attainable in our experiment, in which the sample must be periodically extracted from the coil. The measurements are nevertheless easy to do, the SRM decreasing by only $30 \%$ fifteen minutes after switching off the field.

\section{Conclusion.}

The static magnetic properties of CHAC : Mn $(0.9 \%)$ (susceptibility as a function of frequency and field, remanent magnetizations) are very similar to those observed in other metallic and insulating spin glasses. The case of CHAC : Mn was chosen as an example of a random exchange 1D system. Pure CHAC exhibits intrachain ferromagnetism. Cyclohexylammonium manganese chloride has not been studied to our knowledge, and we did not succeed in growing crystals of this compound ; but it is likely that it would be AFM, like all quasi-1D manganese insulating salts known up to now. We have performed susceptibility experiments on several samples of CHAC : $\mathrm{Mn}$ with different concentrations in order to determine the $\mathrm{Cu}-\mathrm{Mn}$ exchange interaction in the mixed system. These measurements, which are to be published elsewhere, indicate that $J_{\mathrm{Cu}-\mathrm{Mn}}$ is very weak and probably antiferromagnetic. The results of the present paper thus show that a very low amount of exchange randomness is sufficient to induce spin glass properties in 1D systems. From this point of view, a considerable difference appears between 1D and 2D compounds with random exchange. Indeed in the $2 \mathrm{D}$ insulators $\mathrm{K}_{2} \mathrm{Mn}_{1-x} \mathrm{Cr}_{x} \mathrm{Cl}_{4}$ or $\mathrm{K}_{2} \mathrm{Mn}_{1-x} \mathrm{Cu}_{x} \mathrm{~F}_{4}$ which were recently studied [1, 2], spin glass characteristics due to the competition between FM and AFM interactions appear only at mid-range concentrations $(x \simeq 0.4$ to 0.6$)$.

The case of CHAC : Mn is also different from those of the other 1D systems precedently studied. In the boroferrite $\mathrm{FeMgBO}_{4}$, where the $\mathrm{Fe}^{3+}$ ions form zigzag chains, the interactions between nearest neighbours and next nearest neighbours are antiferromagnetic, and about $10 \% \mathrm{Mg}^{2+}$ 
ions replace the iron in the chains [10]. In the pyrochlores $\mathrm{CsMnFeF}_{6}$ and $\mathrm{CsMnNiF}_{6}$, whose structure consists of interconnected chains of fluoride polyhedra including the magnetic ions, large nearest neighbour antiferromagnetic interactions were measured [11]. In both cases, the spin glass-like behaviour is thus due to the presence of frustration and of a large amount of disorder.

Systematic studies of the influence of the $\mathrm{Mn}^{2+}$ impurity concentration on the magnetic properties of CHAC : Mn are in progress and will be published quickly.

\section{References}

[1] Katsumata, K., Nire, T., Tanimoto, M., Yoshizawa, H., Phys. Rev. B 25 (1982) 428;

Katsumata, K., Nire, T., TANimoto, M., Solid State Commun. 43 (1982) 711 ;

Katsumata, K., J. Magn. Magn. Mat. 31-34 (1983) 1435.

[2] Yamada, I., J. Magn. Magn. Mat. 31-34 (1983) 645.

[3] Dupas, C. and Renard, J. P., Phys. Rev. B 18 (1978) 401 ;

Cheikh-Rouhou, A., Dupas, C., Renard, J. P. and Seiden, J., Phys. Rev. B 25 (1982) 3261.

[4] Willett, R. D., Gaura, R. M. and LandeE, C. P., Extended Linear Chain Compounds, Vol. 3, Edited by J. S. Miller (Plenum Publishing Corporation) 1983.

Willett, R. D., Landee, C. P., Gaura, R. M., Swank, D. D., Groenendijk, H. A. and van DuyNEVELDT, A. J., J. Magn. Magn. Mat. 15-18(1980) 1055;

Groenendijk, H. A., van Duyneveldt, A. J. and Willett, R. D., Physica B 101 (1980) 32.

[5] GroenendiJK, H. A., Blöte, H. W. J., van Duyneveldt, A. J., Gaura, R. M., LandeE, C. P. and Willett, R. D., Physica B 106 (1981) 47-58.

[6] Schouten, J. C., VAn Der Geest, G. J., De Jonge, W. J. M. and Kopinga, K., Phys. Lett. A 78 (1980) 398 ;

Kopinga, K., Tinus, A. M. C. and De Jonge, W. J. M., Phys. Rev. B 25 (1982) 4685-4690;

Kopinga, K., Nishihara, H. and De Jonge, W. J. M., J. Magn. Magn. Mat. $31-34$ (1983) 1241.

[7] Tholence, J. L., Solid State Commun. 35 (1980) 113.

[8] Chappert, C., Beauvillain, P., Renard, J. P. and Knorr, K., J. Magn. Magn. Mat. 15-18(1980) 117.

[9] Préjean, J. J., J. Physique Colloq. 39 (1978) C6-907.

[10] Wiedenmann, A. and Burlet, P., J. Physique Colloq. 39 (1978) C6-720;

Wiedenmann, A., Burlet, P., Scheuer, H. and Convert, P., Solid State Commun. 38 (1981) 129.

[11] Alba, M., Hammann, J., Jacoboni, C. and Pappa, C., Phys. Lett. A 89 (1982) 423. 